\title{
Numerical study of an asymmetrically heated rectangular duct with suspended cylinders
}

\author{
Gaurav Kumar Chhaparwal, Ankur Srivastava*, and Ram Dayal \\ Manipal University Jaipur, India 303007
}

\begin{abstract}
An asymmetrically heated (only one side of the duct is heated) solar air heater duct is numerically investigated using openFOAM to study the effect of passive turbulators (suspended cylinders) installed within the boundary layer close to the absorber plate to enhance heat transfer from it. Vortex flow is created behind the suspended cylinders, which disturbs the boundary layer and causes mixing of hot stream lines near the surface with relatively cold stream away from the absorber plate. Diameter of the suspended cylinders and its distance from the absorber plate is varied to study its effect on wall temperature of absorber plate in the turbulent flow regime.
\end{abstract}

$\begin{array}{ll}\text { Nomenclature } \\ \mathrm{f} & \text { Friction factor } \\ \mathrm{Nu} & \text { Nusselt number } \\ \mathrm{W}[\mathrm{mm}] & \text { Width of the rectangular duct } \\ \mathrm{H}[\mathrm{mm}] & \text { Height of the rectangular duct } \\ \mathrm{L}[\mathrm{mm}] & \text { Length of the rectangular duct } \\ \mathrm{AR} & \text { Aspect ratio (W/H) } \\ \mathrm{D}[\mathrm{mm}] & \text { Diameter of the cylinder } \\ \mathrm{S}[\mathrm{mm}] & \text { Transverse distance of cylinder } \\ \mathrm{P}[\mathrm{mm}] & \text { Pitch of two the cylinders } \\ \delta[\mathrm{mm}] & \text { Boundary layer thickness } \\ \mathrm{U}^{+} & \text {Dimensionless free stream velocity } \\ \mathrm{U}[\mathrm{m} / \mathrm{s}] & \text { Free stream velocity } \\ \mathrm{U}_{\mathrm{ref}}[\mathrm{m} / \mathrm{s}] & \text { Reference velocity } \\ \mathrm{y}^{+} & \text {Dimensionless transverse distance } \\ \Delta \mathrm{y}[\mathrm{mm}] & \text { Distance of the first node from the wall } \\ \mathrm{T}[\mathrm{K}] & \text { Temperature of the absorber plate } \\ \mathrm{x}[\mathrm{mm}] & \text { Distance from the leading edge } \\ \mathrm{t}[\mathrm{sec}] & \text { Real time during simulation } \\ \mathrm{Re}_{\mathrm{x}} & \text { Reynolds number at distance } \mathrm{x} \\ \omega & \text { Turbulent Frequency } \\ \rho & \text { Density of the air } \\ \mathrm{U}_{\mathrm{j}} & \text { Free stream velocity in y direction } \\ \mu & \text { Dynamic viscosity }\end{array}$

\footnotetext{
Corresponding author: ankur.srivastava@jaipur.manipal.edu
}

$\begin{array}{ll}\mu_{\mathrm{t}} & \text { Turbulent dynamic viscosity } \\ v & \text { Kinematic viscosity } \\ \varepsilon & \text { Turbulent eddy dissipation } \\ \mathrm{P}_{\mathrm{k}} & \text { Production rate of turbulence }\end{array}$

\section{Introduction}

A solar air heater is a device which uses solar energy to heat up air that is made to pass through its duct. This hot air can be used for various applications such as; cloth dryer, crop dryer, packaging product, room heating etc. Aim of the present study is to improve its poor Thermal-Hydraulic Performance Parameter (THPP).

Absorber plate of the solar air heater absorbs solar radiation and gets heated up. Air blower is used to blow air through a duct, which is covered by absorber plate. Rectangular duct is perfectly insulated except on absorber plate part. Flowing air takes up heat from absorber plate and gets heated mostly though convection mode of heat transfer. But the THPP of such solar air heater is low. This is because convective heat transfer coefficient between absorber plate and air is very low. Many researchers have used different artificial roughness such as: V shape continues inclined and straight ribs, broken ribs, wire mesh, circular ribs etc. to enhance heat transfer. After comprehensive literature review it has been found that $\mathrm{V}$ shape rib is most efficient among all the other type of ribs. All these ribs are engraved on the surface of absorber plate. None of the researchers have used suspended cylinders installed at some distance away from the absorber plate. When air strikes on these cylinders, vortex flow is created behind the face of the cylinders. This vortex flow causes air in the vicinity of absorber plate (which has maximum air temperature) to get mix with relatively cold air in the middle of duct.

The different kinds of roughness, used by researchers have been shown in table 2 (see at the end of the paper). 
Table 2 is represented in graphical form in the fig 1 , fig 2 and fig 3 which shows the variation of friction factor, Nusselt number and THPP at the different combination of e/D, P/e ratios of the different artificial roughness. The behavior of the fluid flow by varying the certain parameters of the ribs or the geometry is not fixed. CFD analysis can be helpful in the predictions of the various parameters of the fluid by changing the geometries very accurately, which is the motive of this project.

Experimental study is common in the field of solar air heater, only few numerical studies have been done so far, to analyze heat transfer enhancement in a solar air heater. The objective of the proposed study is to perform a series of numerical simulations using computational fluid dynamics (CFD) tools to arrive at the optimum configuration for maximum THPP of the solar air heater.

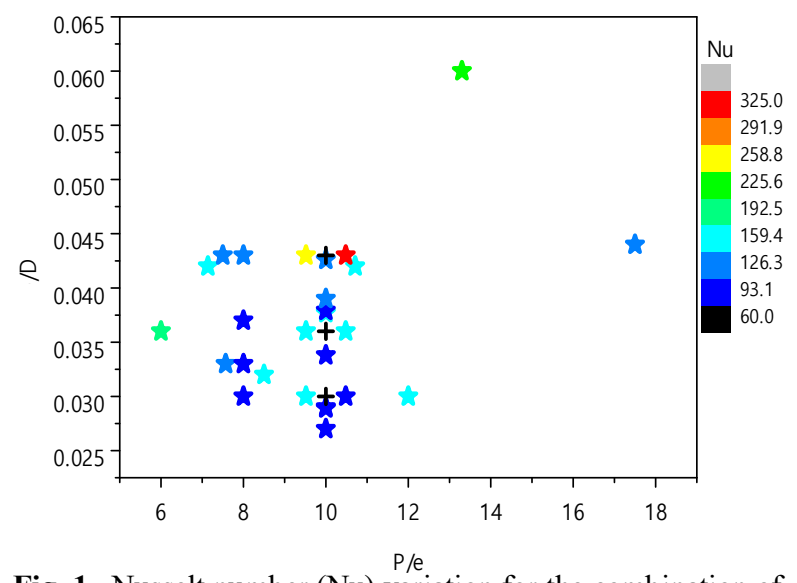

Fig. 1. Nusselt number $(\mathrm{Nu})$ variation for the combination of 'e/D' and 'P/e' of the roughness.

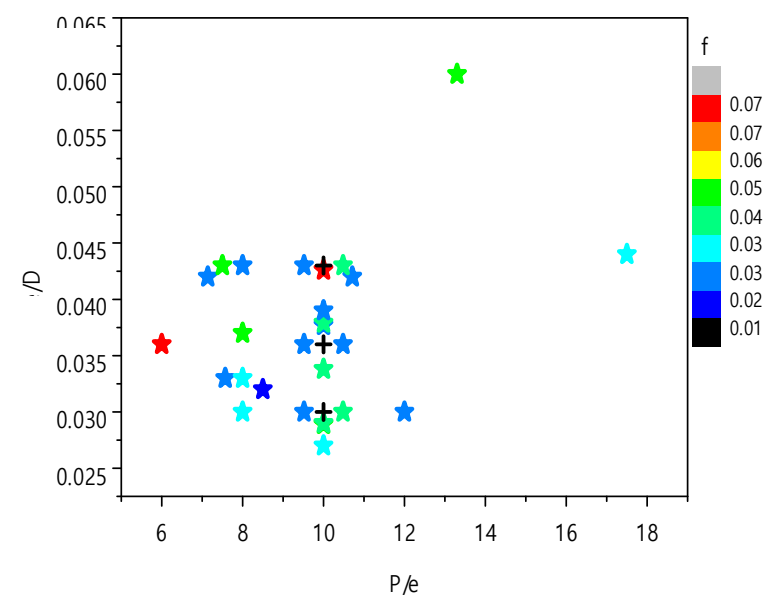

Fig. 2. Friction factor (f) variation for the combination of 'e/D' and ' $\mathrm{P} / \mathrm{e}$ ' parameters of the roughness.

$$
T H P P=\frac{\left[\frac{N u \text { no for roughned duct }}{\text { Nu no for smooth duct }}\right]}{\left[\frac{f \text { for roughned duct }}{f \text { for smooth duct }}\right]^{\frac{1}{3}}}
$$

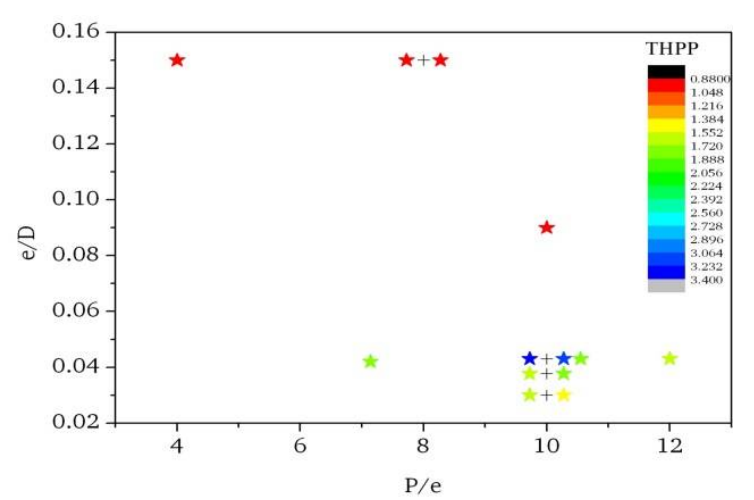

Fig. 3. THPP variation for the combination of ' $\mathrm{e} / \mathrm{D}$ ' and ' $\mathrm{P} / \mathrm{e}$ ' parameters of the roughness.

\section{Modeling}

\subsection{Mathematical modeling}

Three turbulent models; $k-\omega, k-\varepsilon$ and SST were used and results were compared subsequently. And it is found that the $\mathrm{k}-\omega$ model gives the suitable results for our computational domain. It solves two transport equations, one for the turbulent kinetic energy $\mathrm{k}$, and another for the turbulent frequency $\omega$. The stress tensor is computed from the eddyviscosity concept.

$\mathrm{k}$-equation is given by

$\frac{\delta \rho k}{\delta t}+\frac{\delta}{\delta x_{j}}\left(\rho U_{j} k\right)=\frac{\delta}{\delta x_{j}}\left[\left(\mu+\frac{\mu_{t}}{\sigma_{k}}\right) \frac{\delta k}{\delta x_{j}}\right]+P_{k}-\beta^{\prime} \rho \varepsilon \omega+P_{k b}$

$\omega$-equation is given by

$\frac{\delta \rho \omega}{\delta t}+\frac{\delta}{\delta x_{j}}\left(\rho U_{j} \omega\right)=\frac{\delta}{\delta x_{j}}\left[\left(\mu+\frac{\mu_{t}}{\sigma_{\omega}}\right) \frac{\delta \omega}{\delta x_{j}}\right]+\alpha \frac{\omega}{k} P_{k}-\beta^{\prime} \rho \varepsilon \omega^{2}+P_{\omega b}$

In addition to the independent variables, the density $\rho$, and the velocity vector $\mathrm{U}$, are treated as known quantities from the Navier-Stokes method. $\mathrm{k}$ is the production rate of turbulence. The model constants are given by: $\beta{ }^{`}=0.09, \beta=$ $0.075, \alpha=5 / 9, \sigma_{\mathrm{k}}=2, \sigma_{\omega}=2, \mathrm{k}=0.00135, \varepsilon=0.2278125, \omega$ $=1875, \mu_{\mathrm{t}} / \mu=1$

\subsection{Physical model}

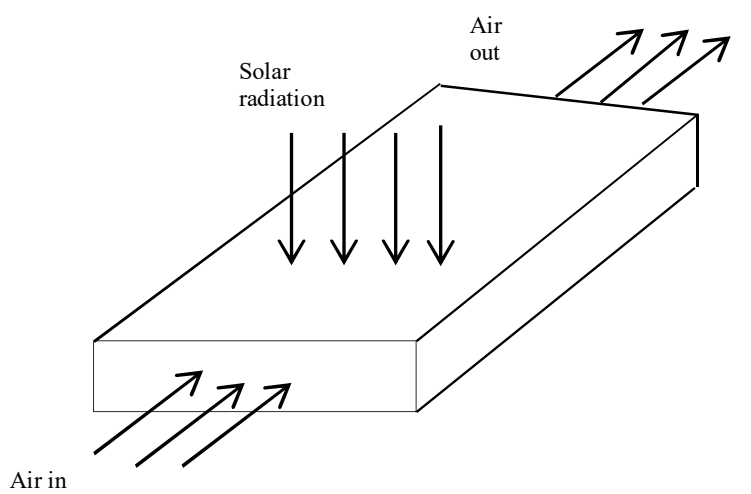

Fig. 4. Schematic diagram of a solar air heater duct.

Fig. 4 shows the schematic diagram of a rectangular duct of a solar air heater to absorb solar radiation and transfer this solar energy to incoming air Solar air heater duct is rectangular in shape. It has four surfaces; two lateral 
surfaces, one upper surface which is known as absorber plate and one lower surface which is called back plate. Width $\mathrm{W}$ and height $b$ both are important design parameters of a solar air heater and decide the pressure loss and the heat transfer in the duct. Reducing height increases heat transfer but pressure loss too in the duct and vice versa.

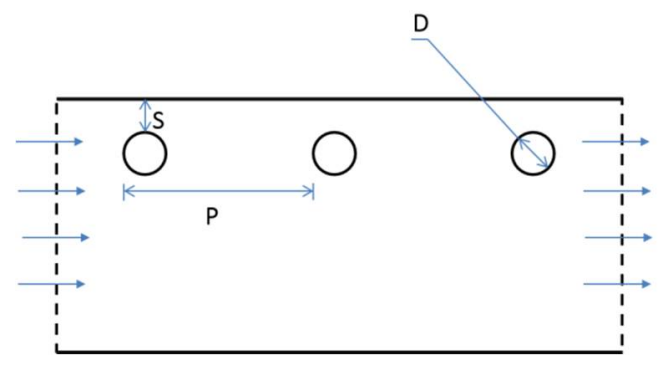

Fig. 5. Cross section diagram of a solar air heater duct along the length.

Fig. 5 shows the cross section of the rectangular duct, but here suspended cylinders are installed in the same duct to exploit the von-Karman effect of vortex flow behind these cylinders. Apart from width W, height $b$, we have, three new important parameters; those are needed to be studied. These parameters are; distance of cylinder from the absorber surface $\mathrm{S}$, distance between centres of any two cylinders, i.e. pitch $\mathrm{P}$ and diameter of the cylinder D.

\subsection{Numerical model}

\subsubsection{Design of experiment}

We limited our study for two parameters only; S and D. All possible different cases, in terms of different combinations of S and D are taken for the study. Preliminary results rejected unfruitful cases and we finally left with only four cases as shown in table 1. Reference for the variation in D and $\mathrm{S}$ is taken from the value of boundary layer thickness $\delta$ $=4.8 \mathrm{~mm}$, at $200 \mathrm{~mm}$ from the leading edge, that can be given by

$$
\frac{\delta}{x}=0.382 R e_{x}^{-1 / 5}
$$

In numerical modeling, 3D domain is avoided due to increase in number of mesh elements that will increase the computing time. 2D geometry and its meshing is done in such a way that it resembles the 3D domain, and the results obtained are within the acceptable limits.

\subsubsection{Geometry}

Geometry is modeled in ANSYS workbench as shown in fig. 6. A 2D domain consists of a circle which is representing cylinder in a plane, whose diameter and distance from the surface vary case to case; therefore four different cases are modelled as given in table 1 .
Table 1. Various possible as per different S and D.

\begin{tabular}{|c|c|c|}
\hline Case & Diameter (D) $\mathbf{( m m )}$ & $\begin{array}{c}\text { Distance from wall (S) } \\
(\mathbf{m m})\end{array}$ \\
\hline Case 1 & 5.0 & 5.0 \\
\hline Case 2 & 5.0 & 2.5 \\
\hline Case 3 & 2.5 & 5.0 \\
\hline Case 4 & 2.5 & 2.5 \\
\hline
\end{tabular}

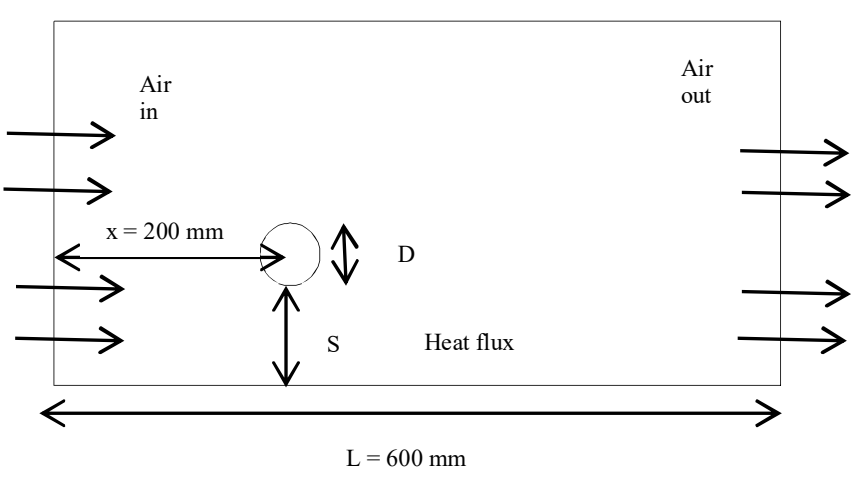

Fig. 6. Schematic diagram of computational domain.

\subsubsection{Meshing}

Geometry is then imported in ICEM CFD meshing software. Here mesh is generated; mesh elements near the cylinder are kept high to observe the von Karman effect of the vortex flow behind the cylinder. We have taken, $\mathrm{y}^{+}=1$, for which $\Delta y=4 \times 10^{-6} \mathrm{~mm}$. Fig 7 shows the overall mesh of the configuration. Fig 8 and fig 9 show the enlarge view meshing near the cylinder boundary.

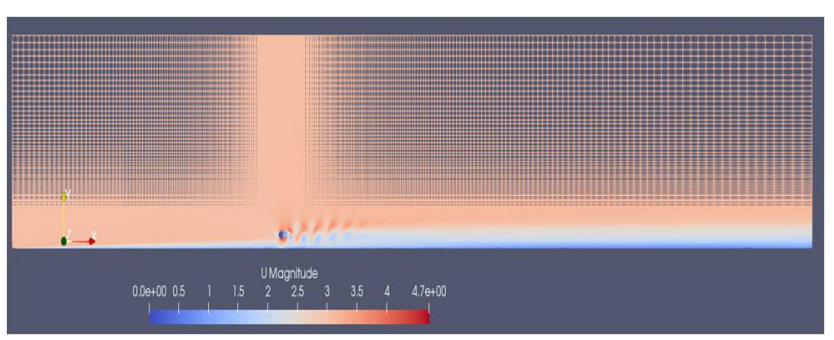

Fig. 7. Overview of the mesh along the length of the flat plate.

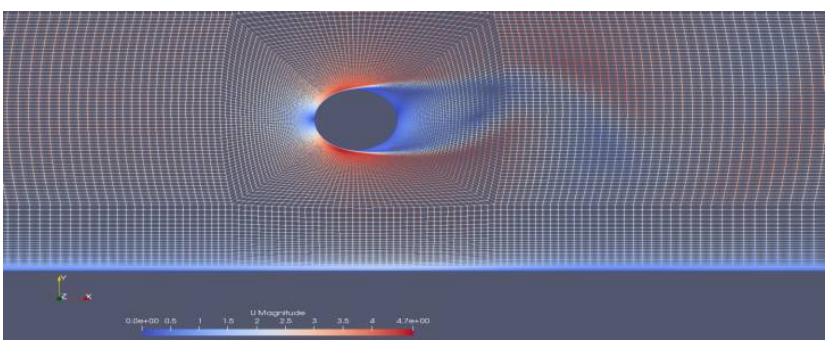

Fig. 8. Mesh shape around the suspended cylinder.

\section{Result analyses}

Mesh created in ICEM CFD is imported in openFOAM. Boundary conditions are applied and problem domain is solved; first for the mesh independency test then for the 
validation of results and finally results are obtained and plotted with the help of PARAVIEW.

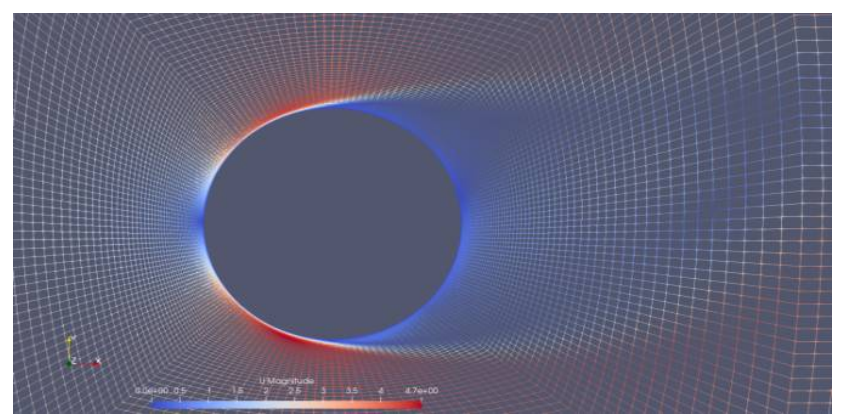

Fig. 9. Enlarged view of mesh around the suspended cylinder.

\subsection{Boundary condition}

2D domain can be treated as a flat plate over which a cylinder is placed $200 \mathrm{~mm}$ from the leading edge. Inlet: turbulent flow is observed after distance of $100 \mathrm{~mm}$ from the leading edge for the critical Reynolds number $5 \times 10^{5}$. Corresponding to this Reynolds number, the input velocity of the air flow is kept $3.0 \mathrm{~m} / \mathrm{s}$. Temperature of the inlet air is $300 \mathrm{~K}$, as room temperature. Outlet: Zero static pressure is assumed, to get fully developed flow. Absorber plate: A constant heat flux of $1000 \mathrm{~W} / \mathrm{m}^{2}$ is applied on the absorber plate. Our aim is to diffuse this heat in the fluid domain to the maximum possible extent. It is assumed that suspended cylinder over the absorber plate doesn't participate in any mode of heat transfer; its only purpose is to create vortex flow.

\subsection{Validation}

For the baseline geometry (without suspended cylinders) and geometry with suspended cylinder, validation of numerical results is conducted. In validation, numerical results are compared with the standard results for $\mathrm{U}^{+}$and $\mathrm{y}^{+}$value as shown in the fig 10 and fig 11. Simulation results performed in OpenFOAM matches satisfactorily with the standard/benchmark results.

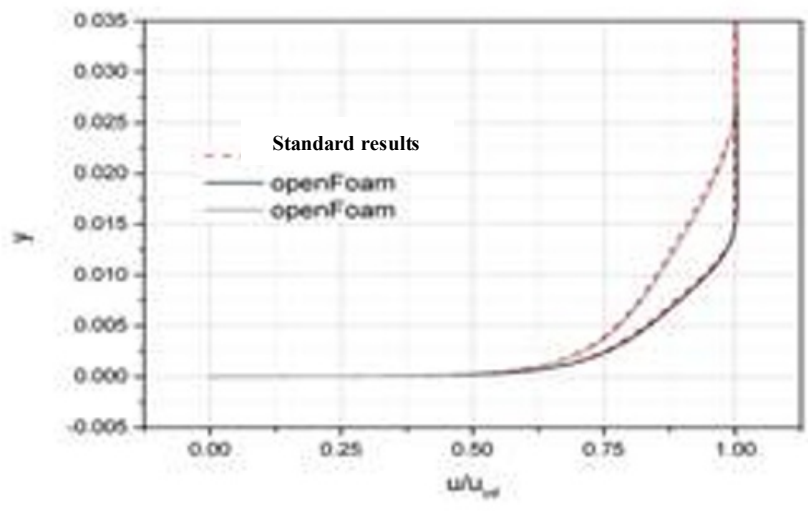

Fig. 10. Comparison of the benchmark result with the OpenFOAM results in terms of variation of $\mathrm{U}^{+}$value with logarithmic $\mathrm{y}^{+}$value.

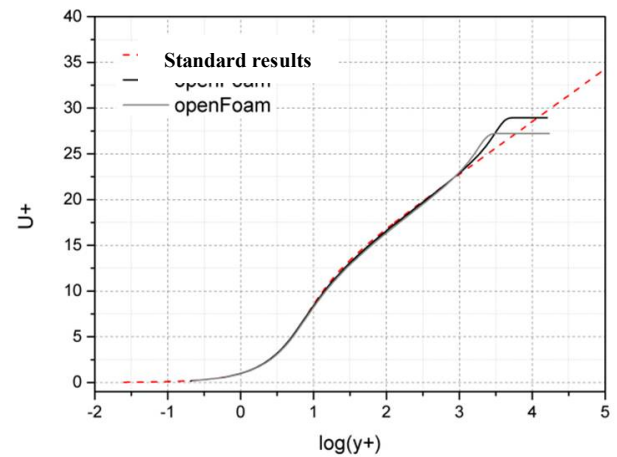

Fig. 11. Comparison of the benchmark result with the OpenFOAM results in terms of variation of $y$ value with free stream velocity.

\subsection{Comparison of different cases}

Different cases are compared with baseline results. Geometry with suspended cylinder showed heat transfer increment over the baseline result.

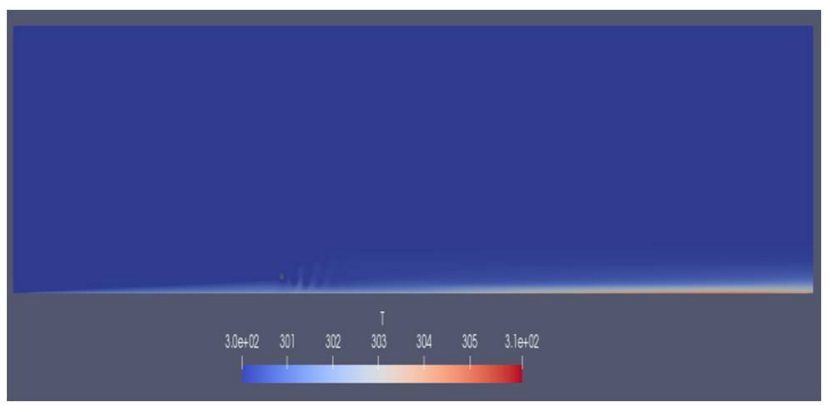

Fig. 12 Temperature profile along the length of the domain for the case 2.

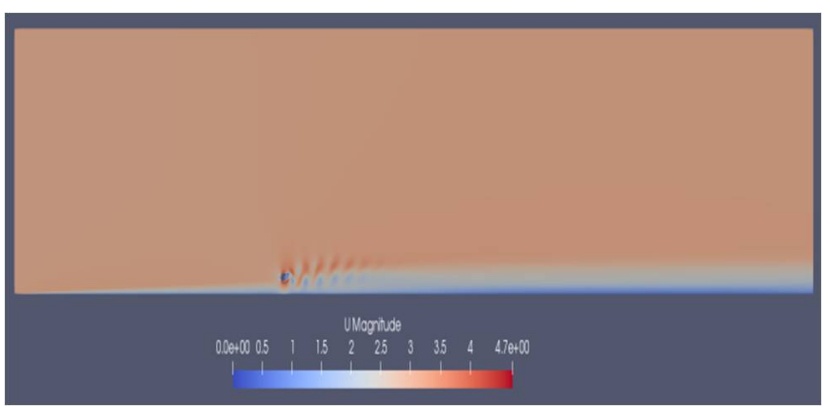

Fig. 13. Velocity profile along the length of the domain for the case 2.

Fig 12 and fig 13 show the pictorial results of change in the velocity and temperature profile, after inclusion of the suspended cylinder for the case 2 . It can be clearly observed with the help of contour plot of velocity and temperature profile that due to presence of cylinder, mixing of hot air near the heated surface with air of relatively lower temperature takes place. Hence the wall temperature of the heated plate decreases behind the cylinders. Similarly, for the case 4, as shown in fig 14 and fig 15, velocity profile and temperature profile show the similar pattern after and before cylinder impediment. But in case 4, the reduction in wall temperature is slightly less the case 2 . Velocity contour in fig 15 shows turbulence for the case 4 than case 2 , similarly darker temperature contour in fig 2 suggest more heat supplied to the streamlines away from the wall/surface. 


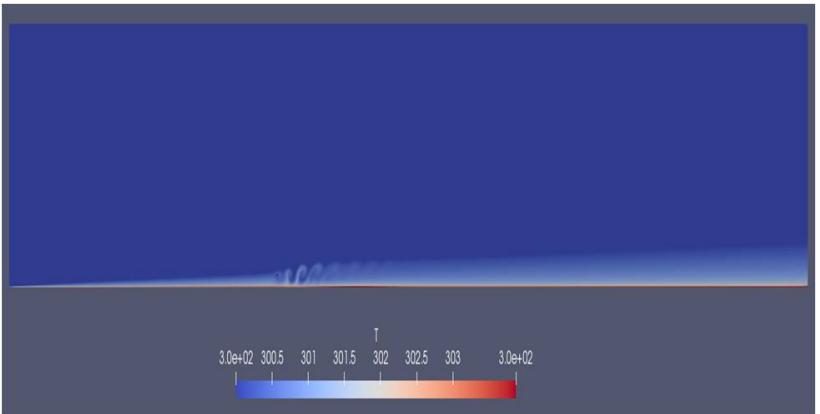

Fig. 14. Temperature profile along the length of the domain for the case 4 .

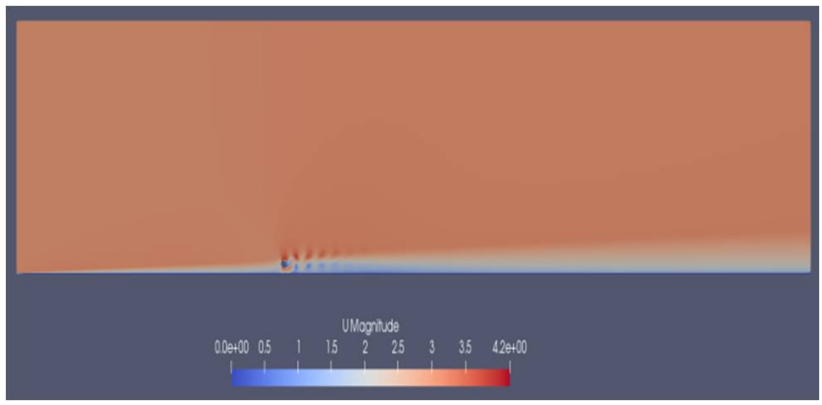

Fig. 15. Velocity profile along the length of the domain for the case 4 .

Fig 16 shows the graphical variation of temperature with time along the length of the duct of the baseline geometry. It shows the transient nature of temperature variation along the along the duct, hence all cases are studied after steady state is achieved. Fig 17 shows the variation of the wall temperature along the length of the duct for different cases, based on positioning of suspended cylinders and change in diameter. Temperature increases as we move from the leading edge of the geometry. But this increment in temperature is more for the baseline geometry than the different cases of suspended cylinder. Temperature increment curve for the baseline geometry is smooth. For the different cases of cylinders, temperature increment curve has abrupt change in slope around the cylinder at a distance of $0.2 \mathrm{~m}$ from the leading edge. Temperature decreases for a moment then it increases again after passing the vortex flow behind the cylinder. But this maximum temperature reached is less then maximum temperature of baseline geometry. Case 4 has minimum rise in the temperature as compared to the other geometries.

In case 4 we have $\mathrm{D}=2.5 \mathrm{~mm}$ and $\mathrm{S}=2.5 \mathrm{~mm}$, both values comes within the boundary layer thickness at that point i.e. $\delta=4.8 \mathrm{~mm}$, hence results are favourable for these values.

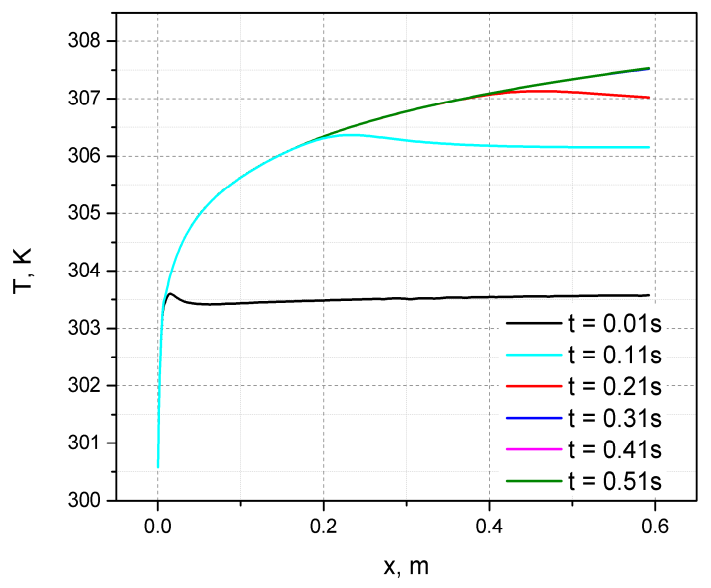

Fig. 16. Temperature variation with time along the length of the SAH duct.

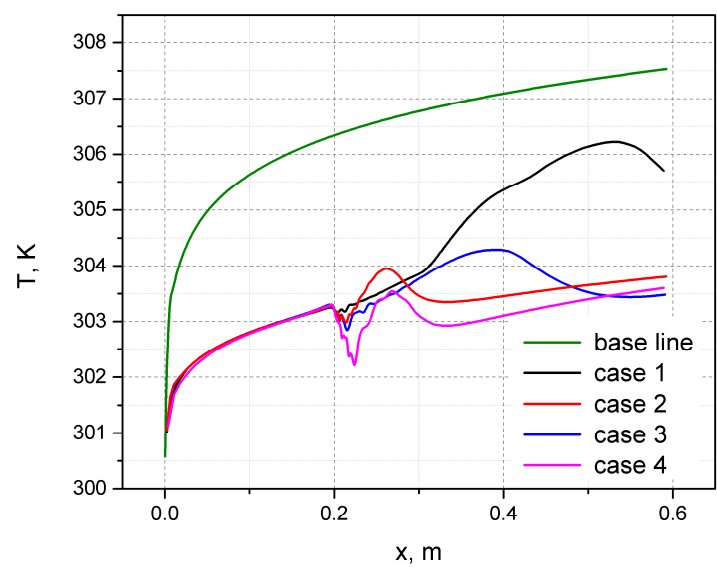

Fig. 17. Temperature variation along the length of SAH duct for different cases.

\section{Conclusions}

Following points can be concluded from the above observation and discussion of this numerical study;

1. Periodic vortex shedding behind the cylinder induces mixing of hot and cold stream, thereby enhancing heat transfer from the heated wall.

2. Positioning of cylindrical turbulator within the boundary layer causes higher heat transfer compared to the case when it is positioned beyond boundary layer thickness.

3. In case of a rectangular duct, it is recommended to place the cylindrical turbulators at adistance beyond entrance length, in order to have higher heat transfer from the heated wall 


\section{Acknowledgements}

We are very thankful to Manipal University Jaipur for providing financial assistance seed grant name MUJ/REGR/1403/11 for this research and lab facility to carry out simulation work.

\section{References}

1. Kumar S, Sharad R.P., Renewable Energy 34 (2009) 1285-1291

2. Bhagoria J.L., Saini J.S., Solanki S.C., Renewable Energy 25 (2002) 341-369

3. Bopche Santosh B., Tandale Madhukar S., International Journal of Heat and Mass Transfer 52 (2009) 2834-2848.

4. Tanda G, International Journal of Heat and Mass Transfer 47 (2004) 229-243

5. Karwa R., Solanki S.C.,International Journal of Heat and Mass Transfer 42(1999) 1597-1665

6. Hans V.S., Saini R.P., Saini R.C., Solar Energy 84 (2010) 898-911

7. Jaurker A.R., Saini J.S., Gandhi B.K., Solar Energy 80 (2006) 895-907

8. Karwa R., Solanki S.C., Saini J.S., Energy 26 (2001) $161-176$

9. Kumar A., Energy Procedia 57 ( 2014 ) 2102 - 2111

10. Patnaik A., Saini R.P., Singal S.K., Renewable Energy 34 (2009) 2914-2922

11. Schlichting, H., 1979, Boundary-Layer, Theory, $7^{\text {th }}$ edition, McGraw-Hill, New York. 
Table 2. Summary of different artificial roughness used by researchers with details of important parameters

\begin{tabular}{|c|c|c|c|c|c|c|c|c|c|}
\hline $\begin{array}{l}\text { S. } \\
\text { No }\end{array}$ & Geometry of the rib & p/e & e/D & $\mathrm{St} / \mathrm{St}_{\mathrm{s}}$ & $\mathrm{Nu} / \mathrm{Nu}_{\mathrm{s}}$ & $\operatorname{Re}$ & $f / f_{s}$ & THPP & Name of the author \\
\hline 1. & Rib- Groove & 6 & 0.0363 & --- & 2.667 & 20000 & 3.333 & 1.785 & A.R.Jaurket et al. \\
\hline 2. & $\begin{array}{c}\text { Multiple V-ribs facing } \\
\text { downward }\end{array}$ & 12 & 0.043 & --- & 4.629 & 20000 & 27.38 & 1.56 & V.S.Hans et al. \\
\hline 3. & $\begin{array}{c}\text { Transverse } \\
\text { Continuous ribs }\end{array}$ & 8 & 0.15 & --- & 2.1 & 40000 & 12.1428 & 0.914 & G.Tanda et al. \\
\hline 4. & Transverse broken ribs & 4 & 0.15 & --- & 2.5 & 40000 & 18.57 & 0.944 & G.Tanda et al. \\
\hline 5. & Broken v-ribs & 8 & 0.15 & --- & 2.02 & 40000 & 10.71 & 0.916 & G.Tanda et al. \\
\hline 6. & V-shaped continuous & 10 & 0.03 & --- & 1.579 & 13500 & 1.381 & 1.418 & Aharwal and lanjewar et al. \\
\hline 7. & V-shaped with gap & 10 & 0.03 & --- & 1.842 & 13500 & 1.534 & 1.601 & Aharwal and lanjewar et al. \\
\hline 8. & Inverted u-shaped ribs & 6.67 & 0.0398 & -- & 2.917 & 18000 & 3.833 & 1.864 & Bopche and Tandale et al. \\
\hline 9. & Transverse wedge ribs & 7.57 & 0.033 & -- & 2.3 & 18000 & 3 & 1.596 & Bhagoria et al. \\
\hline 10. & Chamfered repeated ribs & 4.58 & 0.0321 & 2 & --- & 17000 & 2.625 & 1.45 & Karwa et al. \\
\hline 11. & Arc-shaped ribs & 10 & 0.0426 & -- & 1.474 & 18000 & 1.744 & 1.225 & Kumar and saini et al. \\
\hline 12. & Dimple shaped ribs & 10 & 0.0289 & -- & 2.57 & 11500 & 4 & 1.62 & Saini and verma at el. \\
\hline 13. & Inclined continuous with gap & 10 & 0.0377 & -- & 2.58 & 18000 & 2.9 & 1.809 & K.R.Aharwal et al. \\
\hline 14. & Inclined continuous & 10 & 0.0377 & -- & 2.3 & 18000 & 2.5 & 1.695 & K.R.Aharwal et al. \\
\hline 15. & V-shaped rib & 10 & 0.043 & -- & 2 & 20000 & 3 & 1.387 & A.Kumar et al. \\
\hline 16. & Multi V-shaped rib & 10 & 0.043 & -- & 5 & 20000 & 4 & 3.15 & A.Kumar et al. \\
\hline 17. & Multi V-shaped with gap & 10 & 0.043 & -- & 5.5 & 20000 & 4.25 & 3.395 & A.Kumar et al. \\
\hline 18. & Transverse rectangular ribs & 10 & 0.09 & -- & 2.12 & 40000 & 14 & 0.88 & G.Tanda et al. \\
\hline 19. & Transverse square ribs & 7.14 & 0.042 & -- & 2.78 & 18000 & 3.557 & 1.824 & A.S.Yadav et al. \\
\hline 21. & Equilateral triangular rib & 14.29 & 0.021 & -- & 2.13 & 18000 & 2.357 & 1.565 & A.Yadav and Bhagoria et al. \\
\hline 22. & Equilateral triangular rib & 10 & 0.03 & -- & 2.766 & 18000 & 2.714 & 1.983 & A.Yadav and Bhagoria et al. \\
\hline 23. & Equilateral triangular rib & 7.14 & 0.042 & -- & 3.276 & 18000 & 3.286 & 2.204 & A.Yadav and Bhagoria et al. \\
\hline 20. & Chamfered repeated ribs & 4.58 & 0.0441 & -- & 2.2 & 11000 & 3.8 & 1.6021 & R.Karwa et al. \\
\hline
\end{tabular}

